\begin{abstract}
Iranica
Abstracta Iranica Revue bibliographique pour le domaine irano-aryen

Volume 34-35-36 | 2017

Comptes rendus des publications de 2011-2013
\end{abstract}

\title{
Julio Bendezu-Sarmiento (ed.). L'archéologie française en Asie centrale. Nouvelles recherches et enjeux socioculturels
}

\section{Rémy Boucharlat}

\section{(2) OpenEdition \\ Journals}

Édition électronique

URL : http://journals.openedition.org/abstractairanica/42047

DOI : 10.4000/abstractairanica.42047

ISSN : 1961-960X

Éditeur :

CNRS (UMR 7528 Mondes iraniens et indiens), Éditions de l'IFRI

Référence électronique

Rémy Boucharlat, « Julio Bendezu-Sarmiento (ed.). L'archéologie française en Asie centrale. Nouvelles recherches et enjeux socioculturels », Abstracta Iranica [En ligne], Volume 34-35-36 | 2017, document 9, mis en ligne le 30 juillet 2017, consulté le 27 septembre 2020. URL : http://journals.openedition.org/ abstractairanica/42047 ; DOI : https://doi.org/10.4000/abstractairanica.42047

Ce document a été généré automatiquement le 27 septembre 2020.

Tous droits réservés 


\title{
Julio Bendezu-Sarmiento (ed.). L'archéologie française en Asie centrale. Nouvelles recherches et enjeux socioculturels
}

\author{
Rémy Boucharlat
}

\section{RÉFÉRENCE}

Julio Bendezu-Sarmiento (ed.). L'archéologie française en Asie centrale. Nouvelles recherches et enjeux socioculturels. Paris-Bichkek-Kaboul, diff. De Boccard, 2013, 638 p., ill. (Cahiers d'Asie centrale 21-22)

1 Ce gros volume de 33 contributions, bien présenté et largement illustré, tente de rendre compte des activités de plusieurs missions archéologiques conjointes, avec une partie française et une partie turkmène, ouzbèke, kazakhe, tadjike, afghane. À la différence de la structure fédérative allemande, l'Eurasien Abteilung de l'Institut archéologique allemand, mais comme par exemple les équipes italiennes, les missions partie française sont issues de différentes équipes de chercheurs du CNRS surtout et de l'Université, mais toutes bénéficient pour une part de leur budget de subventions du Ministère français des Affaires étrangères.

2 La coopération en Afghanistan est très ancienne - la Délégation archéologique française dans ce pays a été créée en 1922, mais elle fut fermée entre 1980 et 2002 tandis que les activités en Asie centrale débutèrent en 1989 par la création d'une Mission archéologique française en Asie Centrale (MAFAC). Elle créait ainsi un support qui officialisait une coopération établie entre les archéologues français et leurs collègues soviétiques depuis deux décennies, Cette coopération s'est poursuivie et étendue après 1991 avec les institutions archéologiques de chacune des nouvelles républiques d'Asie centrale indépendantes. Les contributions de R. Besenval pour les « 
années noires » et de Ph. Marquis pour les quinze dernières années rendent compte de l'activité française en Afghanistan. Pour l'Asie centrale, on verra celles de S. Gorshenina pour la coopération et de H.-P. Francfort plus spécifiquement pour l'histoire de la MAFAC.

3 À côté des périodes antérieures au I ${ }^{\mathrm{er}}$ mill. av. J.C., représentées par huit articles, les périodes depuis le milieu du $\mathrm{I}^{\mathrm{er}}$ mill. av. J.-C. jusqu'à l'époque médiévale sont traitées spécialement par une douzaine de contributions. Pour l'âge du Fer et l'époque achéménide, on note la reprise des recherches après les fouilles de l'époque soviétique puis russe sur le site de Padayatak tépé, près de Shahr-i Sabz, en Sogdiane, Ouzbékistan actuel, sur lequel les constructions achéménides reconnues sont encore très limitées (J. Lhuillier et M. Hasanov), ainsi que les travaux sur les niveaux supérieurs de Ulug Dépé au Turkménistan (O. Lecomte). C'est la dernière et plus importante occupation du site sur 5 hectares, constituée des vestiges d'une véritable ville au réseau viaire bien identifié, protégée par une enceinte. Elle comprend aussi des édifices monumentaux et dans la ville haute une citadelle dont le plan évoque de près celui des «forts » du Zagros, Nush-i Jan, Godin Tepe, Tepe Ozbaki, en Iran, et Tell Ghubba dans le Kurdistan irakien, mais celle d'Ulug est bien antérieure, puisqu'elle est datée au tournant des $\mathrm{II}^{\mathrm{e}}$ et $\mathrm{I}^{\mathrm{er}}$ millénaires, et sa réoccupation du $\mathrm{IX}^{\mathrm{e}}$ au VII ${ }^{\mathrm{e}}$ siècle.

4 La période hellénistique jusqu'aux Kouchans est bien représentée. Ce sont les travaux dans la région de Termez et particulièrement sur ce site, occupé de l'époque hellénistique jusqu'aux Mongols (P. Leriche depuis 1993). Colonie militaire séleucide puis gréco-bactrienne, ensuite capitale des Yue Tche, puis des Kouchans, elle et un centre important du bouddhisme, la ville a livré un palais, plusieurs édifices à caractère religieux et une puissante fortification. Mais beaucoup reste à découvrir. La céramique de toutes les périodes est étudiée par J.-B. Houal et le décor architectural en pierre à l'époque kouchane par M.-O. Perou.

5 Le site de Samarkand lui-même, où se déroulent les fouilles récentes depuis 1989, n'est représenté que par la présentation d'un grenier à sel d'époque grec (C. Baratin et L. Martinez-Sève), mais pour les environs, on trouvera un résumé des recherches sur Koktepe (C. Rapin et M. Isamiddinov). La période II, à partir du VII ${ }^{e}$ ou VI ${ }^{\mathrm{e}}$ s. av. J.-C., est marquée par des ensembles architecturaux fortifiés, sur plate-forme, l'un à vocation économico-politique, l'autre religieuse. La période achéménide Koktepe III suit sans hiatus mais est marquée par la construction de bâtiments dans des aires jusque-là découvertes et l'occupation déborde les anciennes limites. Après des occupations plus modestes, au tournant de l'ère chrétienne, une des plateformes est transformée en kourgane pour la sépulture d'une aristocrate qualifiée de scytho-sarmate. Les nécropoles de Yangi-rabat et Akdzhar-tepe, de 30 et 9 kourganes respectivement, n'ont pas toutes été fouillées (J. Vallée-Raewsky). Ce sont soit des fosses simples soit, pour trois d'entre eux, des kourganes pourvus d'un dromos. Malgré le pillage général, la céramique retrouvée permet de les dater entre le début du III ${ }^{\mathrm{e}} \mathrm{s}$. et le milieu du II $\mathrm{e}$ s. av. $\mathrm{J} .-\mathrm{C}$, rectifiant les datations proposées naguère ( $\mathrm{I}^{\mathrm{er}} \mathrm{s}$. avant et $\mathrm{I}^{\mathrm{er}} \mathrm{s}$. après $\mathrm{J} . \mathrm{C}$. $)$.

6 Pour l'époque islamique, P. Siméon présente la production de Hulbuk, capitale du Huttal, au SE du Tadjikistan entre le $\mathrm{IX}^{\mathrm{e}}$ et le $\mathrm{XI}^{\mathrm{e}}$ s., poursuivant celle des périodes antérieures depuis la fin de l'âge du Bronze par B. Lyonnet qui envisage l'ensemble de Sogdiane. Dans l'oasis de Boukhara, depuis 2010, deux premières saisons de prospections et de fouilles se sont déroulés sur la ville de Paykend, à $45 \mathrm{~km}$ au SO de Boukhara (R. Rante et A. Raimkulov). Ils marquent la reprise de travaux antérieurs. Les 
recherches se concentrent sur les $\mathrm{X}^{\mathrm{e}}$ et $\mathrm{XI}^{\mathrm{e}}$ siècles dans la citadelle et l'un des šahrestān$\mathrm{s}$, mais ont touché aussi les niveaux inférieurs qui remontent à la fondation de la ville au IV de n.è., qui était alors proche de la frontière nord de l'empire sassanide.

7 Le tableau des activités archéologiques comprend également la présentation des recherches d'archéozoologie ( $M$. Mashkour), de paléobotanique (M. Tengberg) et d'hydrologie (E. Fouache et al. mais dans ce cas sur des régions de l'Iran oriental), l'évolution des techniques céramiques (A. Dupont-Delaleuf), qui toutes concernent principalement les époques antérieures à l'âge du Fer. Figurent également une contribution sur l'apport de la géomatique aux travaux de terrain. Le volume est complété par la présentation des indispensables opérations de protection et conservation que nécessitent la mise au jour des vestiges : conservation et restauration des objets principalement, à Ulug dépé et Gonur dépé (E. Ottenwelter), la restauration de peintures murales à Kazakl'i-yaktan/Akchakhan-Kala, que fouille une mission australo-ouzbèke, et Afrasiab d'époque qarakhanide (G. Fray et M. Reutova), enfin de l'architecture de terre à Ulug dépé (c. Sadozaï).

\section{AUTEURS}

\section{RÉMY BOUCHARLAT}

UMR 5133 CNRS-Université de Lyon 